\title{
New Approaches for the Uses of 2-Cyanomethyl Thiazole in the Synthesis of Fused Heterocyclic Derivatives with Anti-Tumor Activities
}

\author{
Wagnat W. Wardakhan \\ National Organization for Drug Control \& Research P. O 29, \\ Cairo, Egypt.
}

\begin{abstract}
2 -(4,5-DIHYDRO-4-oxothiazol-2-yl)acetonitrile (1) reacted with 22 acetylcoumarin (2) to give the 2-(4,5-dihydro-4-oxothiazol-2-yl)3-(2-oxo-2H-chromen-3-yl)but-2-enenitrile (3). The reactivity of the latter product towards aromatic aldehydes $4 \mathrm{a}-\mathrm{d}$, cyanomethylene reagents $6 \mathrm{a}, \mathrm{b}$, aryl diazonium salts $10 \mathrm{a}-\mathrm{d}$, was studied to give products that were evaluated against the three cancer cells namely breast adenocarcinoma (MCF-7), non-small cell lung cancer (NCI-H460) and CNS cancer (SF-268). Some of them have high inhibitory effect towards three cell lines and compounds 14 and 16 were found to be more active than the standard on NCI-H460 and SF-268.
\end{abstract}

Keywords: Thiazole, Coumarin, Pyrazole, Pyridazine and Anti-tumor.

Thiazoles play a prominent role in nature. For example, the thiazolium ring present in vitamin B1 serves as an electron sink and its coenzyme form is important for the decarboxylation of $\alpha$-keto acids ${ }^{(1)}$. Various pesticides possessing a thiazole nucleus are well known in agriculture. Large numbers of thiazole derivatives have emerged as active pharmaceutical ingredients in several drugs for their potential antiinflammatory $^{(2)}$, anti-tumor ${ }^{(3)}$ anti-hyperlipidemic ${ }^{(4)}$, anti-hypertensive ${ }^{(5)}$ and several other biological properties ${ }^{(6)}$. Besides, thiazoles are also synthetic intermediates and common substructures in numerous biologically active compounds ${ }^{(7-13)}$.

\section{Chemistry}

\section{Results and Discussion}

In this work we describe the uses of 2-(4,5-dihydro-4-oxothiazol-2yl)acetonitrile(1), which was obtained earlier ${ }^{(14,15)}$, through the reaction of malononitrile with thioglycollic acid in acetic acid. Compound 1 reacts with acetophenone (2) in the presence of ammonium acetate at $140^{\circ} \mathrm{C}$ to give the Knoevenagel condensation product 3. The structure of compound 3 was based on analytical and spectral data. Thus, the ${ }^{1}$ HNMR spectrum showed a singlet at $\delta \square 2.89$ ppm corresponding for the $\mathrm{CH}_{3}$ group, a singlet at $\delta 5.65 \mathrm{ppm}$ corresponding to the $\mathrm{CH}_{2}$, and a multiplet at $\delta$ 7.30-7.39 for phenyl protons.<smiles>N#CC1=NC(=O)CS1</smiles>

1<smiles>CC(=O)c1cc2ccccc2oc1=O</smiles>

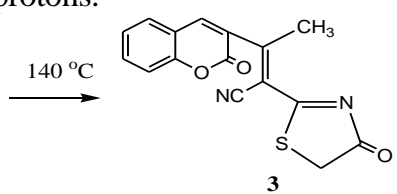

E-mail: wagnatward@ hotmail.com 
The reaction of compound 3 with aromatic aldehydes was studied to give arylidine derivatives with potential biological activities. Thus, compound 3 reacts with either benzaldehyde (4a), 4-chlorobenzaldehyde (4b), 4-methoxy-benzaldehyde (4c) or salicylaldehyde (4d), to give in each case a single product for them either structures 5a-d were assigned. Structures of the latter products were based on analytical and spectral data (see experimental section).

3

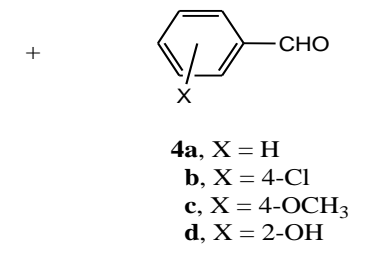

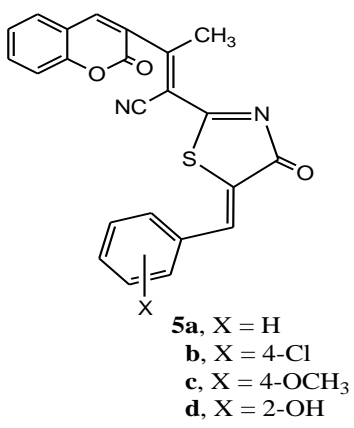

The reaction of compound 5a with either malononitrile (6a) or ethyl cyanoacetate (6b) gave the polyfunctionally substituted pyrano[2,3-d]thiazole derivatives $8 \mathrm{a}, \mathrm{b}$. The reaction took place via the intermediate formation of 7a,b (Scheme 1). Structures of compounds $8 \mathrm{a}$ and $8 \mathrm{~b}$ were established on the basis of analytical and spectral data (see experimental section). Further confirmation for structures $8 \mathrm{a}, \mathrm{b}$ was obtained through the synthesis of these compounds using another reaction routes. Thus, the reaction of compound 3 with either $\alpha$-cyano cinmamonitrile (9a) or ethyl $\alpha$ cyanocinmamate (9b) (Scheme 1) in the presence of 1,4-dioxan and a catalytic amount of triethylamine gave the same polyfunctionally substituted pyrano[2,3d]thiazole derivatives $8 \mathrm{a}$ and $8 \mathrm{~b}$, respectively (finger print IR spectrum, m.p. and mixed m.p.).

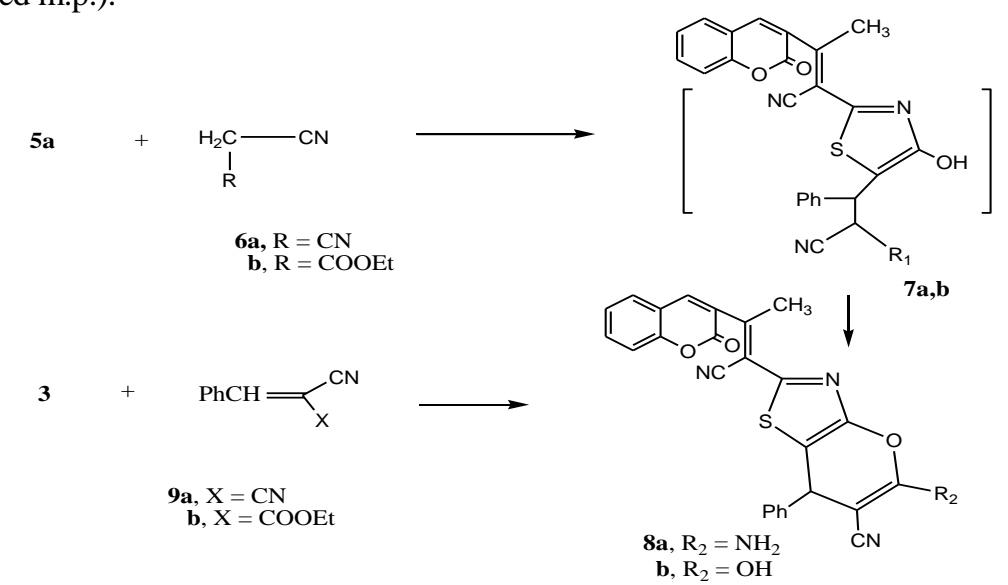

The reaction of compound 3 with either benzenediazonium chloride (10a), 4chlorobenzenediazonium chloride (10b), 4-methybenzenediazonium chloride

Egypt. J. Chem. 54, No. 2 (2011) 
(10c) or 4-methoxybenzenediazonium chloride (10d) gave aryl-hydrazone derivatives 11a-d respectively. The analytical and spectral data of the latter products are consistent with the proposed structures (see experimental section).

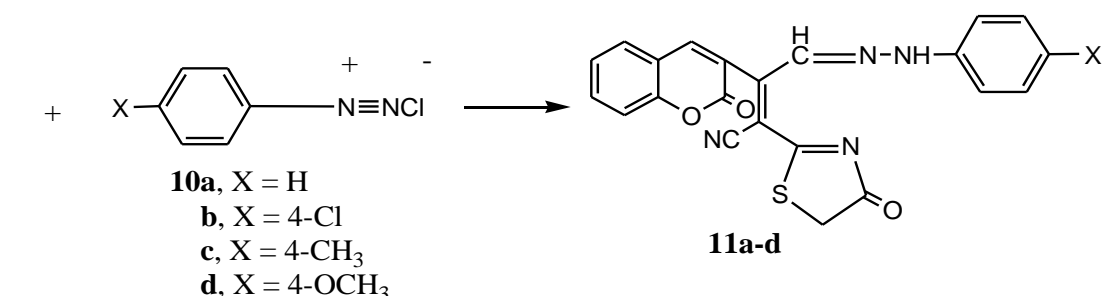

\section{Antitumor activity}

Material, methods \& reagents

Fetal bovine serum (FBS) and L-glutamine, were from Gibco Invitrogen Co. (Scotland, UK). RPMI-1640 medium was from Cambrex (New Jersey, USA). Dimethyl sulfoxide (DMSO), doxorubicin, penicillin, streptomycin and sulforhodamine B (SRB) were from Sigma Chemical Co. (Saint Louis, USA). Samples: Stock solutions of selected compounds from 3-11a-d were prepared in DMSO and kept at $-20{ }^{\circ} \mathrm{C}$. Appropriate dilutions of the compounds were freshly prepared just prior the assays. Final concentrations of DMSO did not interfere with the cell growth.

\section{Cell cultures}

Three human tumor cell lines, MCF-7 (breast adenocarcinoma), NCI-H460 (non-small cell lung cancer) and SF-268 (CNS cancer) were used. MCF-7 was obtained from the European Collection of Cell Cultures (ECACC, Salisbury, UK) and NCI-H460 and SF-268 were kindly provided by the National Cancer Institute (NCI, Cairo, Egypt). They grow as monolayer and routinely maintained in RPMI-1640 medium supplemented with $5 \%$ heat inactivated FBS, $2 \mathrm{mM}$ glutamine and antibiotics (penicillin $100 \mu / \mathrm{ml}$, streptomycin $100 \mu \mathrm{g} / \mathrm{ml}$ ), at $37^{\circ} \mathrm{C}$ in a humidified atmosphere containing $5 \% \mathrm{CO}_{2}$. Exponentially growing cells were obtained by plating $1.5 \times 10^{5}$ cells/ml for MCF-7 and SF-268 and $0.75 \times$ $10^{4}$ cells $/ \mathrm{ml}$ for NCI-H460, followed by $24 \mathrm{hr}$ of incubation. The effect of the vehicle solvent (DMSO) on the growth of these cell lines was evaluated in all the experiments by exposing untreated control cells to the maximum concentration $(0.5 \%)$ of DMSO used in each assay.

\section{Effect on the growth of human tumor cell lines}

The effect of selected compounds from the newly synthesized products 3 11a-d was evaluated on the in vitro growth of three human tumor cell lines representing different tumor types, namely, breast adenocarcinoma (MCF-7), non-small cell lung cancer (NCI-H460) and CNS cancer (SF-268), after a continuous exposure of $48 \mathrm{hr}$. The results are summarized in Table 1. 
All the compounds were able to inhibit the growth of the human tumor cell lines in a dose-dependent manner (data not shown). The 2-pyridylthiazole derivative 14 and the 2-thiophenylthiazole derivatives 16 showed the best results, exhibiting an equivalent potency in all the three tumor cell lines which is still much lower than the gram positive control doxorubicin, On the other hand, compounds 5a, 8a, 11b, 11d and 12a showed moderated growth inhibitory effect. Comparing the activities of $12 \mathrm{a} \& \mathrm{~b}$ it is observed that the N-phenyl group in $12 \mathrm{a}$ presents a stronger growth inhibitory effect than the N-p-chloroaryl substituent in $12 \mathrm{~b}$ although the results in NCI-H460 cell line, are comparable. It is clear from Table 1 that some compounds like $5 \mathrm{~d}, 11 \mathrm{a}, 11 \mathrm{c}$ and $12 \mathrm{~d}$ showed very low activity towards certain cell line, MCF-7 and moderate activity towards other cell lines.

TABLE 1. Effect of compounds selected on the growth of three human tumor cell lines.

\begin{tabular}{|c|c|c|c|}
\hline \multirow[t]{2}{*}{ Compound } & \multicolumn{3}{|c|}{ GI50 $\left(\mu \square \mathrm{mol} \mathrm{L}^{-1}\right)$} \\
\hline & MCF-7 & \multirow{2}{*}{$\frac{\text { NCI-H460 }}{16.0 \pm 4.8}$} & SF-268 \\
\hline 3 & $30 \square \pm 2.9$ & & $28.0 \pm 2.8$ \\
\hline $\begin{array}{l}\mathbf{5 a} \\
3.9\end{array}$ & $33 \square \pm 11.6$ & $29.3 \pm 4.2$ & $38 \pm$ \\
\hline $5 \mathbf{b}$ & $0.4 \pm 0.02$ & $2.0 \pm 0.8$ & $0.9 \pm 0.6$ \\
\hline $\begin{array}{l}\mathbf{5 c} \\
12.6\end{array}$ & $60 \square \pm 12.2$ & $48.0 \pm 6.4$ & $58 \pm$ \\
\hline $5 d$ & $30.4 \pm 0.02$ & $22.4 \pm 6.2$ & $11.2 \pm 2.8$ \\
\hline $8 \mathbf{a}$ & $18.6 \pm 4.6$ & $10.5 \pm 1.8$ & $22.4 \pm 2.8$ \\
\hline $8 b$ & $12.8 \pm 6.6$ & $18.2 \pm 4.2$ & $20.2 \pm 4.5$ \\
\hline $11 \mathrm{a}$ & $0.1 \pm 0.08$ & $0.7 \pm 0.06$ & $0.4 \pm 0.06$ \\
\hline $11 b$ & $20.1 \pm 2.4$ & $36.2 \pm 2.4$ & $38.0 \pm 2.7$ \\
\hline $11 \mathrm{c}$ & $30.0 \pm 2.6$ & $40.2 \pm 4.5$ & $20.2 \pm 4.8$ \\
\hline 11d & $66.2 \pm 12.8$ & $48.1 \pm 4.6$ & $55.8 \pm 8.3$ \\
\hline Doxorubicin & $0.04 \pm 0.008$ & $0.09 \pm 0.008$ & $0.09 \pm 0.007$ \\
\hline $\begin{array}{l}\text { Doxorubicin } \\
0.09 \pm 0.007\end{array}$ & 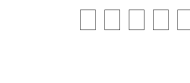 & $4 \pm 0.008$ & $0.09 \pm 0.008$ \\
\hline
\end{tabular}

Results are given in concentrations that were able to cause $50 \%$ of cell growth inhibition $\left(\mathrm{GI}_{50}\right)$ after a continuous exposure of $48 \mathrm{hr}$ and show means \pm SEM of three-independent experiments performed in duplicate.

\section{Experimental}

All melting points are uncorrected. IR spectra were recorded for $(\mathrm{KBr})$ discs on Pye Unicam SP-1000 Spectrophotometer ${ }^{1}$ HNMR Spectra were measured on a Varian EM-390-200 MHz and Bruker AVANCE DRX-500-300 MHz in $\mathrm{CD}_{3} \mathrm{SOCD}_{3}$ as solvent using TMS as internal standard and chemical shifts are expressed as $\delta .{ }^{13}$ CNMR spectra were measured on Bruker AVANCE DRX-500. 
Analytical data were obtained from Micro Analytical Data Unit at Cairo University, Giza, Egypt.

2-(4,5-Dihydro-4-oxothiazol-2-yl)-3-(2-oxo-2H-chomen-3-yl)but-2-enenitrile (3)

To a dry mixture of compound $1(0.01 \mathrm{~mol}, 1.87 \mathrm{~g}), 3$-acetylcoumarin $(1.88 \mathrm{~g}$, $0.01 \mathrm{~mol})$ was added in the presence of ammonium acetate $(2.0 \mathrm{~g})$. The reaction mixture was heated in an oil bath $\left(\right.$ at $\left.140{ }^{\circ} \mathrm{C}\right)$ for $1 \mathrm{hr}$, then left to cool. The solid product formed after boiling in ethanol, was collected by filtration. Yellow crystals from ethanol, yield $\mathrm{g}(80 \%)$; mp $188-190{ }^{\circ} \mathrm{C}$. IR: v 3387-3232 (OH), $2986\left(\mathrm{CH}_{3}\right), 2223(\mathrm{CN}), 1708,1688(2 \mathrm{CO}), 1636(\mathrm{C}=\mathrm{C}) \mathrm{cm}^{-1} . \mathrm{MS}: \mathrm{m} / \mathrm{z}(\%)=$ $310\left(100, \mathrm{M}^{+}\right)$. ${ }^{1} \mathrm{HNMR}: \delta 2.72\left(\mathrm{~s}, 3 \mathrm{H}, \mathrm{CH}_{3}\right), 5.68\left(\mathrm{~s}, 2 \mathrm{H}, \mathrm{CH}_{2}\right), 6.02(\mathrm{~s}, 1 \mathrm{H}$, coumarin H-4), 7.28-7.37 (m, 9H, $\left.\mathrm{C}_{6} \mathrm{H}_{5}, \mathrm{C}_{6} \mathrm{H}_{4}\right)$. Calculated for $\mathrm{C}_{16} \mathrm{H}_{10} \mathrm{~N}_{2} \mathrm{O}_{3} \mathrm{~S}$ (310.33): C, 61.93; H, 3.25; N, 9.03; S, 10.33. Found: C, 62.02; H, 3.36; N, 9.21; S, 10.60 .

\section{Synthesis of compounds $5 a-d$ \\ General procedure}

An equimolar amount of either compound $3(0.01 \mathrm{~mol}, 3.10 \mathrm{~g})$ in ethanol (30 $\mathrm{ml})$ containing pipridine $(0.5 \mathrm{ml})$ either benzaldehyde $(0.01 \mathrm{~mol}, 1.06 \mathrm{ml})$, pchlorobenzaldehyde $(1.40 \mathrm{~g}, 0.01 \mathrm{~mol})$, p-methoxy-benzaldhyde $(1.36 \mathrm{~g}, 0.01$ $\mathrm{mol})$, or salicylaldehyde $(1.22 \mathrm{~g}, 0.01 \mathrm{~mol})$ was added. The reaction mixture was heated under reflux for $3 \mathrm{hr}$ then poured into ice/water mixture containing few drops of hydrochloric acid. The solid product formed, in each case, was collected by filtration.

2-(5-Benzylidene-4,5-dihydro-4-oxothiazol-2-yl)-3- (2-oxo-2H- chomen-3-yl) but- 2- enenitrile (5a)

Yellow crystals from ethanol, yield $2.78 \mathrm{~g}(70 \%)$, mp 231-234 ${ }^{\circ} \mathrm{C}$. IR: v 3066 (CH aromatic), 2986, $2869(\mathrm{CH} 3), 1690,1685(2 \mathrm{CO}), 1633(\mathrm{C}=\mathrm{C}) \mathrm{cm}^{-1}$. ${ }^{1} \mathrm{H}$ NMR: $\delta 2.89\left(\mathrm{~s}, 3 \mathrm{H}, \mathrm{CH}_{3}\right), 6.23(\mathrm{~s}, 1 \mathrm{H}$, coumarin $\mathrm{H}-4), 7.28-7.33(\mathrm{~m}, 15 \mathrm{H}$, $\left.2 \mathrm{C}_{6} \mathrm{H}_{4}, \mathrm{C}_{6} \mathrm{H}_{5}, \mathrm{CH}\right)$. Calculated for $\mathrm{C}_{23} \mathrm{H}_{14} \mathrm{~N}_{2} \mathrm{O}_{3} \mathrm{~S}$ (398.43): $\mathrm{C}, 69.33 ; \mathrm{H}, 3.54 ; \mathrm{N}$, 7.03; S, 8.05. Found: C, 69.77; H, 3.79; N, 7.38; S, 8.11.

2-(5-4-Chlorobenzylidene-4,5- dihydro-4- oxothiazol-2-yl)-3- (2-oxo- $2 \mathrm{H}-$ chomen -3-yl) but-2- enenitrile (5b)

Yellow crystals from ethanol, yield $2.59 \mathrm{~g}(60 \%) ; \mathrm{mp} 190-192{ }^{\circ} \mathrm{C}$. IR: v 3060 (CH aromatic), $2937\left(\mathrm{CH}_{3}\right), 1692,1685(2 \mathrm{CO}), 1620(\mathrm{C}=\mathrm{C}) \mathrm{cm}^{-1} .{ }^{1} \mathrm{HNMR} \delta=$ $2.77\left(\mathrm{~s}, 3 \mathrm{H}, \mathrm{CH}_{3}\right), 6.20\left(\mathrm{~s}, 1 \mathrm{H}\right.$, coumarib H-4), 7.30-7.40 (m, $14 \mathrm{H}, 2 \mathrm{C}_{6} \mathrm{H}_{4}, \mathrm{C}_{6} \mathrm{H}_{5}$, $\mathrm{CH})$. Calculated for $\mathrm{C}_{23} \mathrm{H}_{13} \mathrm{ClN}_{2} \mathrm{O}_{3} \mathrm{~S}(432.03) \mathrm{C}, 63.82 ; \mathrm{H}, 3.03 ; \mathrm{N}, 6.47 ; \mathrm{S}, 7.41$. Found: C, 63.82; H, 3.24; N, 6.73; S, 7.82.

2-(5-4-methyoxybenzylidene -4,5-dihydro -4-oxothiazol-2-yl) -3-(2-oxo- $2 \mathrm{H}$ chomen -3-yl) but-2-enenitrile (5c)

Yellow crystals from ethanol; yield $3.29 \mathrm{~g}(80 \%)$; mp 180-183 ${ }^{\circ} \mathrm{C}$. IR: v $3054\left(\mathrm{CH}\right.$ aromatic), $2970\left(\mathrm{CH}_{3}\right), 1688,1684(2 \mathrm{C}=\mathrm{O}), 1631(\mathrm{C}=\mathrm{C}) \mathrm{cm}^{-1}$. ${ }^{1}$ HNMR: $\delta \quad 2.88,3.32\left(2 \mathrm{~s}, 6 \mathrm{H}, 2 \mathrm{CH}_{3}\right), 6.20(\mathrm{~s}, 1 \mathrm{H}$, coumarin $\mathrm{H}-4), 7.26-7.40(\mathrm{~m}$, 
$\left.14 \mathrm{H}, 2 \mathrm{C}_{6} \mathrm{H}_{4}, \mathrm{Ph}, \mathrm{CH}\right)$. Calculated for $\mathrm{C}_{24} \mathrm{H}_{16} \mathrm{~N}_{2} \mathrm{O}_{3} \mathrm{~S}$ (412.46): C, 69.89; H, 3.91; N, 6.79; S, 7.77. Found: C, 69.87; H, 4.03; N, 6.82; S, 8.02.

2-(5-4-methoxybenzylidene -4,5-dihydro -4-oxothiazol-2-yl) -3-(2-oxo-2Hchomen -3-yl) but-2-enenitrile (5d)

Yellow crystals from ethanol, yield $2.99 \mathrm{~g}(70 \%)$; mp 170-173 ${ }^{\circ} \mathrm{C}$. IR: v 3566-3233 $(\mathrm{OH}), 3060\left(\mathrm{CH}\right.$ aromatic), $2960\left(\mathrm{CH}_{3}\right), 1690,1686(2 \mathrm{CO}), 1631$ $(\mathrm{C}=\mathrm{C}) \mathrm{cm}^{-1}$. ${ }^{1} \mathrm{HNMR}: \delta$ 2.86, $3.11\left(2 \mathrm{~s}, 6 \mathrm{H}, 2 \mathrm{CH}_{3}\right), 6.21(\mathrm{~s}, 1 \mathrm{H}$, cumarin $\mathrm{H}-4)$, 7.28-7.34 (m, $\left.14 \mathrm{H}, \mathrm{C}_{6} \mathrm{H}_{5}, 2 \mathrm{C}_{6} \mathrm{H}_{4}, \mathrm{CH}\right)$. Calculated for $\mathrm{C}_{24} \mathrm{H}_{16} \mathrm{~N}_{2} \mathrm{O}_{4} \mathrm{~S}$ (428.46): $\mathrm{C}$, 67.28, H, 3.76: N, 6.54; S, 7.48. Found: C, 67.53; H, 3.61; N, 7.59; S, 7.362.

Synthesis of compounds $8 a, b$

General procedure

Method A : To a solution of compound 5a (0.01 mol, $3.98 \mathrm{~g})$ in ethanol (30 $\mathrm{mL})$ containing triethylamine $(0.5 \mathrm{ml})$, either malononitrile $(0.66 \mathrm{~g}, 0.01 \mathrm{~mol})$ or ethyl cyanoacetate $(1.13 \mathrm{~g}, 0.01 \mathrm{~mol})$ was added, the reaction mixture was heated under reflux for $4 \mathrm{hr}$. The solid product formed upon pouring onto ice/water mixture was collected by filtration.

Method B : To a solution of compound $3(0.01 \mathrm{~mol}, 3.10 \mathrm{~g})$ in ethanol (30 $\mathrm{mL})$ containing triethylamine $(0.5 \mathrm{ml})$, either $\alpha$-cyanocinnamonitrile $(1.70 \mathrm{~g}$, $0.01 \mathrm{~mol})$ or ethyl $\alpha$-cyanocinnamate $(1.97 \mathrm{~g}, 0.01 \mathrm{~mol})$ was added. The reaction mixture was heated under reflux for $5 \mathrm{hr}$ then the reaction mixture was poured onto ice/water mixture containing few drops of hydrochloric acid and the formed solid product was collected by filtration.

5-Ami ol-cyano-2- (2-oxo-2H- chomen-3-yl) prop-1-enyl) -7-phenyl -7Hpyrano [2,3-d] thiazole-6-carbonitrile ( $8 a)$

Orange crystals from ethanol, yield $2.78 \mathrm{~g}(60 \%)$; mp $220-222^{\circ} \mathrm{C}$. IR: v 3455-3320 $\left(\mathrm{NH}_{2}\right), 3050\left(\mathrm{CH}\right.$ aromatic), $2946\left(\mathrm{CH}_{3}\right), 2222(\mathrm{CN}), 1688(\mathrm{CO})$, $1564(\mathrm{C}=\mathrm{C}) .{ }^{1} \mathrm{HNMR}: \delta \quad 2.87\left(\mathrm{~s}, 3 \mathrm{H}, \mathrm{CH}_{3}\right), 5.58\left(\mathrm{~s}, 2 \mathrm{H}, \mathrm{NH}_{2}\right), 6.03($ Coumarin $\mathrm{H}-$ 4), 7.24-7.37 (m, $10 \mathrm{H}, \mathrm{C}_{6} \mathrm{H}_{5}, \mathrm{C}_{6} \mathrm{H}_{4}$, pyran $\left.\mathrm{H}-4\right)$. Calculated for $\mathrm{C}_{26} \mathrm{H}_{16} \mathrm{~N}_{4} \mathrm{O}_{3} \mathrm{~S}$ (464.50): C, 67.23; H, 3.47; N, 12.06; S, 6.90. Found: C, 67.24; H, 3.37; N, $12.085 ; \mathrm{S}, 7.16$.

1-Cyano-2-(2-oxo-2H- chomen-3-yl) prop-1-enyl)-5-hydroxy-7-phenyl-7Hpyrano $[2,3-d]$ thiazole -6-carbonitrile $(8 b)$

Yellow crystals from ethanol, yield $3.58 \mathrm{~g}(77 \%)$; mp 147-149 ${ }^{\circ} \mathrm{C}$. IR: v 3566-3132 $(\mathrm{OH}), 3053\left(\mathrm{CH}\right.$ aromatic), $2966\left(\mathrm{CH}_{3}\right), 2220(\mathrm{CN}), 1678(\mathrm{CO}), 1632$ $(\mathrm{C}=\mathrm{C}) \mathrm{cm}^{-1} .{ }^{1} \mathrm{HNMR}: \delta 2.83\left(\mathrm{~s}, 3 \mathrm{H}, \mathrm{CH}_{3}\right), 6.24$ (coumarin $\left.\mathrm{H}-4\right), 7.29-7.41(\mathrm{~m}$, $10 \mathrm{H}, \mathrm{C}_{6} \mathrm{H}_{5}, \mathrm{C}_{6} \mathrm{H}_{4}$, pyrane $\left.\mathrm{H}-4\right), 9.22(\mathrm{~s}, 1 \mathrm{H}, \mathrm{OH})$. Calculated for $\mathrm{C}_{26} \mathrm{H}_{15} \mathrm{~N}_{3} \mathrm{O}_{4} \mathrm{~S}$ (465.48): C, 67.09; H, 3.25; N, 9.03; N, 9.03; S, 6.89. Found: C, 67.32; H, 3.51; N, 8.79; N, 8.94; S, 7.03. 
Synthesis of compounds 11a-d

General Procedure

To a cold solution $\left(0-5^{\circ} \mathrm{C}\right)$ of compound $3(2.89 \mathrm{~g}, 0.01 \mathrm{~mol} \mathrm{~g})$ in acetic acid/ethanol (1:4) $(50 \mathrm{ml})$ containing sodium hydroxide $(5 \mathrm{ml}, 10 \%)$ either benzenediazonium chloride $(0.01 \mathrm{~mol}), 4$-chlorobenzene- diazonium chloride (0.01 mol), 4-methylbenzenediazonium chloride $(0.01 \mathrm{~mol})$ or 4-methoxybenzenediazonium chloride $(0.01 \mathrm{~mol})$ [prepared by adding sodium nitrite $(0.02$ mol, $1.38 \mathrm{~g})$ solution to a cold solution $\left(0-5^{\circ} \mathrm{C}\right)$ of aniline $(0.93 \mathrm{~g}, 0.01 \mathrm{~mol}), 4-$ chloroaniline $(1.27 \mathrm{~g}, 0.01 \mathrm{~mol}$,$) , 4-methylaniline (1.07 \mathrm{~g}, 0.01 \mathrm{~mol})$ or 4 methoxyaniline $(1.23 \mathrm{~g}, 0.01 \mathrm{~mol})$ containing the appropriate amount of hydrochloric acid with continuous stirring] was added with stirring. The reaction mixture was left at $0-5^{\circ} \mathrm{C}$ for $4 \mathrm{hr}$ and the formed solid product, in each case, was collected by filtration.

4-(2-(4-Phenyl)- hydrazono) -2-(4,5-dihydro -4-oxothiazol -2-yl)-3-(2-oxo2H-chomen -3-yl) but-2-enenitrile (11a)

Red crystals from ethanol, yield $3.47 \mathrm{~g}(84 \%)$; mp 220-223 ${ }^{\circ} \mathrm{C}$. IR: v 3473$3331(\mathrm{NH}), 3055\left(\mathrm{CH}\right.$ aromatic), 1691, $1688(2 \mathrm{CO}), 1632(\mathrm{C}=\mathrm{C}) \mathrm{cm}^{-1} .{ }^{1} \mathrm{HNMR}$ : $\delta 5.41\left(\mathrm{~s}, 2 \mathrm{H}\right.$, thiazole $\left.\mathrm{CH}_{2}\right), 6.04(\mathrm{~s}, 1 \mathrm{H}, \mathrm{CH}=\mathrm{N}), 6.27(\mathrm{~s}, 1 \mathrm{H}$, coumarin $\mathrm{H}-4)$, 7.29- $7.37\left(\mathrm{~m}, 9 \mathrm{H}, \mathrm{C}_{6} \mathrm{H}_{5}, \mathrm{C}_{6} \mathrm{H}_{4}\right), 8.40(\mathrm{~s}, 1 \mathrm{H}, \mathrm{NH})$. Calculated for $\mathrm{C}_{22} \mathrm{H}_{14} \mathrm{~N}_{4} \mathrm{O}_{3} \mathrm{~S}$ (414.44): C, 63.76; H, 3.40; N, 13.52; S, 7.74. Found: C, 63.84; H, 3.16; N, $13.62 ; \mathrm{S}, 7.84$.

4-(2-(4-chlorophenyl)-hydrazono)-2-(4,5-dihydro-4-oxothiazol-2-yl)-(2-oxo2H-chomen-3-yl)but-2-enenitrile (11b)

Orange powder from ethanol, yield $3.58 \mathrm{~g}(80 \%)$, mp 196-199 ${ }^{\circ} \mathrm{C}$. IR: v $3448-$ $3338(\mathrm{NH}), 3052$ (CH aromatic), 1688, 1668 (2 CO), $1638(\mathrm{C}=\mathrm{C}) \mathrm{cm}^{-1} .{ }^{1} \mathrm{HNMR}$ :

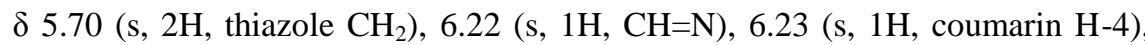
7.29-7.35 (m, $\left.8 \mathrm{H}, 2 \mathrm{C}_{6} \mathrm{H}_{4}\right), 9.40(\mathrm{~s}, 1 \mathrm{H}, \mathrm{NH})$. Calculated for $\mathrm{C}_{22} \mathrm{H}_{13} \mathrm{ClN}_{4} \mathrm{O}_{3} \mathrm{~S}$ (228.88): C, 58.87; H, 2.92; N, 12.48; S, 7.14. Found: C, 58.67; H, 3.08; N, $12.68 ; \mathrm{S}, 7.09$

4-(2-(4-mehylyphenyl)- hydrazono)-2-(4,5- dihydro-4-oxothiazol-2-yl) -3-(2oxo-2H-chomen-3-yl)but-2-enenitrile (11c)

Brown crystals form ethanol, yield $2.57 \mathrm{~g}(60 \%)$; mp $188-192^{\circ} \mathrm{C}$. IR: v 3439$3325(\mathrm{NH}), 3062\left(\mathrm{CH}\right.$ aromatic), $2955\left(\mathrm{CH}_{3}\right), 1688,1680(2 \mathrm{CO}), 1631(\mathrm{C}=\mathrm{C})$ $\mathrm{cm}^{-1} .{ }^{1} \mathrm{HNMR}: \delta 3.24\left(\mathrm{~s}, 3 \mathrm{H}, \mathrm{CH}_{3}\right), 5.45\left(\mathrm{~s}, 2 \mathrm{H}\right.$, thiazole $\left.\mathrm{CH}_{2}\right), 6.01(\mathrm{~s}, 1 \mathrm{H}$, $\mathrm{CH}=\mathrm{N}), 6.25(\mathrm{~s}, 1 \mathrm{H}$, coumarin $\mathrm{H}-4), 7.29-7.37\left(\mathrm{~m}, 8 \mathrm{H}, 2 \mathrm{C}_{6} \mathrm{H}_{4}\right), 8.44(\mathrm{~s}, 1 \mathrm{H}, \mathrm{NH})$. Calculated for $\mathrm{C}_{23} \mathrm{H}_{16} \mathrm{~N}_{4} \mathrm{O}_{3} \mathrm{~S}$ (428.46): C, 64.47; H, 3.76; N, 13.08; S, 7.48. Found: C, 64.31; H, 3.79; N, 13.15; S, 7.58.

4-(2-(4- mehoxyphenyl) -hydrazono)-2-(4,5- dihydro-4- oxothiazol -2-yl)-3phenylbut-2-enenitrile (11d) 
Red crystals from ethanol, yield $3.06 \mathrm{~g}$ (69 \%); mp 148 ${ }^{\circ} \mathrm{C}$. IR: v 3446-3322 $(\mathrm{NH}), 3058$ (CH aromatic), 2989 ( $\mathrm{CH}$ aromatic), $2988\left(\mathrm{CH}_{3}\right), 1685,1683(2 \mathrm{CO})$, $1622(\mathrm{C}=\mathrm{C}) \quad \mathrm{cm}^{-1} .{ }^{1} \mathrm{HNMR}: \delta 3.02\left(\mathrm{~s}, 3 \mathrm{H}, \mathrm{CH}_{3}\right), 5.62\left(\mathrm{~s}, 2 \mathrm{H}, \mathrm{CH}_{2}\right), 6.20(\mathrm{~s}, 1 \mathrm{H}$, $\mathrm{CH}=\mathrm{N}), 6.26(\mathrm{~s}, 1 \mathrm{H}$, coumarin $\mathrm{H}-4), 7.30-7.48\left(\mathrm{~m}, 8 \mathrm{H}, 2 \mathrm{C}_{6} \mathrm{H}_{5}\right), 8.96(\mathrm{~s}, 1 \mathrm{H}, \mathrm{NH})$. Calculated for $\mathrm{C}_{23} \mathrm{H}_{16} \mathrm{~N}_{4} \mathrm{O}_{4} \mathrm{~S}$ (444.46): C, 62.15; H, 3.63; N, 12.61; S, 7.21. Found: C, 62.28; H, 3.58; N, 12.79; S; 7.42.

\section{References}

1. Sondhi, M.S., Singh, N., Johar M. and Kumar, A., Synthesis, anti-inflammatory and analgesic ativities and evaluation of some mono, bi and tricyclic pyrimidine derivatives. Bioorg. \& Med. Chem. 13, 6158-6166 (2005).

2. Yang, Z., Li., Q. and Qian, X., Novel thiazonaphthalimides as efficient antitumor and DNA photocleaving agents: effects of intercalation, side chains and substituents groups. Bioorg. \& Med. Chem. 13, 4864-4870 (2005).

3. Turan-Zitouni, G.Z.A., Kaplancikli, M.T., Chevallet Y.P. and Kaya D., Synthesis and antimicrobial activity of 4-phenyl/ cyclohexyl-5-(1-phenoxyethyl)-3[N-(2-thiazolyl) acetamido] thio-4H-1,2,4-triazole derivatives. Eur. J. Med. Chem. 40, 607-613(2005).

4. Li, Z., Yang, Q. and Qian, X., Novel heterocyclic family of phenyl naphthothiazole carboxamides derived from naphthalimides: synthesis, antitumor evaluation, and DNA photocleavage. Bioorg. \& Med. Chem. 13,3149-3155 (2005).

5. Narayana, B., Vijaya Raj, K. K., Ashalatha, B. V., Kumari, N. S. and Sarojini, B. K., Synthesis of some new 5-(2-substituted-1,3-thiazol-5-yl)-2-hydroxy benzamides and their 2-alkoxy derivatives as possible antifungal agents. Eur. J. Med. Chem. 39, 867-872 (2004).

6. Vicini, P. L., Geronikaki, A., Incerti M., Busonera, B., Poni, G., Cabras, C.A. and Colla, P., Synthesis and biological evaluation of benzo[d] isothiazole, benzothiazole and thiazole Schiff bases. Bioorg. \& Med. Chem. 11, 4785-4789 (2003).

7. Tomasz, K. O. and Boduszek, B., A brief perspective on catalysis from its origins and at the threshold of the $21^{\text {st }}$ century. Tetrahedron, 66, 8661-8666 (2010).

8. Al-Sarawy, A.A., Fouda, A.S. and Shehab El-Dein, W.A., Some thiazole derivatives as corrosion inhibitors for carbon steel in acidic medium. Desalination 229, 79-293 (2008)

9. Bhattacharya, P. J., Leonard, T. and Roy, K., Exploring QSAR of thiazole and thiadiazole derivatives as potent and selective human adenosine A3 receptor antagonists using FA and GFA techniques. Bioorg. Med. Chem. 13, 1159-1165 (2005).

10. Yu, H., Shao, L. H. and Fang, J., Synthesis and biological activity research of novel ferrocenyl-containing thiazole imine derivatives. J. Oranomet. Chem. 692, 991996 (2007).

Egypt. J. Chem. 54, No. 2 (2011) 
11. Bondock, S., Khalifa, W. and Fadda, A. A., Synthesis and antimicrobial evaluation of some new thiazole, thiazolidinone and thiazoline derivatives starting from 1chloro-3,4-dihydronaphthalene-2-carboxaldehyde. Euro. J. Med. Chem. 42, 948- 954 (2007).

12. Nettekoven, M., Guba, W., Neidhart, W., Mattei, P., Pflieger, P., Roche, O. and Taylor, S., Isomeric thiazole derivatives as ligands for the neuropeptide Y5 receptor. Bioorg \& Med. Chem. Lett. 15, 3446-3449 (2005).

13. Mansour, S. A., Mahmoud, S. B., Al-qasoumi, S. I. and Horab, M. M., Antibreast cancer activity of some novel 1,2-dihydropyridine, thiophene and thiazole derivatives. Euro. J. Med.Chem. 46, 137-141 (2011).

14. Elnagdi, M. H., Elmoghayer, M.R., Hammam A. G. and Khallaf, S. A., The reaction of malononitrile with thioglycolic acid. A novel procedure for the synthesis of thiazolone derivatives. J. Heterocycl. Chem. 16, 1541-1546 (1979).

15. Elnagdi, M.H., Khalifa, M.A., Ibrahim, M. K. and Elmoghayer, M. R., Synthesis of new azaindenes and pyrazole derivatives. J. Heterocycl. Chem. 8, 877-889 (1981).

(Received 22/8/2011; accepted 8/9/2011)

اتجاهات حديثة لاستخدامات مركب r بـ سيانو ثيازول لتحضير مركبات عضوية غير متجانسة الحلقة كمضادات لاتبات للأورام

وجنات وهبة وردخان

هيئة الرقابة و البحوث وردان الدوائية ص ـ ب ـ و ب القاهرة - مصر.

مشتق الثيازول ا تفاعل مع مركب بـ أستيل كيومارين ليعطى المركب ساز

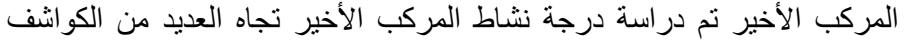

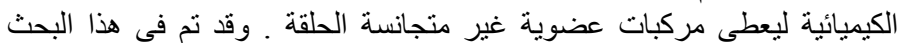

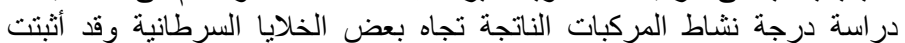

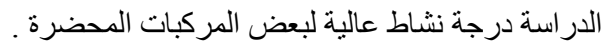

